\title{
Dissociation Of Working Memory from Decision Making within the Human Prefrontal Cortex
}

\author{
Antoine Bechara, ${ }^{1,2}$ Hanna Damasio,, ${ }^{1,3}$ Daniel Tranel, ${ }^{1}$ and Steven W. Anderson ${ }^{1}$ \\ ${ }^{1}$ Department of Neurology, Division of Behavioral Neurology and Cognitive Neuroscience, and ${ }^{2}$ Department of Anatomy \\ and Cell Biology, University of lowa College of Medicine, lowa City, lowa, 52242, and ${ }^{3}$ The Salk Institute of Biological \\ Studies, La Jolla, California, 92186
}

\begin{abstract}
We tested the hypothesis that cognitive functions related to working memory (assessed with delay tasks) are distinct from those related to decision making (assessed with a gambling task), and that working memory and decision making depend in part on separate anatomical substrates. Normal controls ( $n=$ $21)$, subjects with lesions in the ventromedial (VM) $(n=9)$ or dorsolateral/high mesial (DL/M) prefrontal cortices $(n=10)$, performed on (1) modified delay tasks that assess working memory and (2) a gambling task designed to measure decision making. VM subjects with more anterior lesions $(n=4)$ performed defectively on the gambling but not the delay task. VM subjects with more posterior lesions $(n=5)$ were impaired on
\end{abstract}

both tasks. Right DL/M subjects were impaired on the delay task but not the gambling task. Left DL/M subjects were not impaired on either task. The findings reveal a cognitive and anatomic double dissociation between deficits in decision making (anterior VM) and working memory (right DL/M). This presents the first direct evidence of such effects in humans using the lesion method and underscores the special importance of the VM prefrontal region in decision making, independent of a direct role in working memory.

Key words: decision making; working memory; dorsolateral prefrontal cortex; orbitofrontal cortex; delay tasks; gambling tasks; disinhibition; control of interference
Studies in nonhuman primates have shown that lesions of the dorsolateral (DL) prefrontal cortex give rise to severe impairments in working memory (Goldman-Rakic, 1987, 1992). This has been found in several experiments using a variety of delay task procedures. All of them have one feature in common: a temporal gap between a stimulus and a response, i.e., a need to maintain in temporary memory stores a particular stimulus. Such findings are consistent with findings in humans with DL lesions who exhibit impairments in working memory, as defined by Baddeley (1992). They are also consistent with functional neuroimaging studies in humans supporting a role for the DL cortex in working memory (Jonides et al., 1993; Petrides et al., 1993; McCarthy et al., 1994; D'Esposito et al., 1995a; Smith et al., 1995; Cohen et al., 1997; Courtney et al., 1997). Although these studies suggest that the DL cortex is necessary for working memory, the question of whether the DL cortex is necessary for decision making, defined as the ability to select an advantageous response from among an array of available options (Damasio et al., 1991; Damasio, 1994), has not been addressed.

On the other hand, studies in humans with bilateral damage of the ventromedial (VM) prefrontal cortex show that VM subjects develop severe impairments in decision making, as defined above (Eslinger and Damasio, 1984; Grafman et al., 1990; Damasio et al., 1991; Shallice and Burgess, 1991; Damasio, 1994), but their working memory appears normal on standard clinical neuropsychology tests (Grafman et al., 1990; Anderson et al., 1991; Tranel

Received Aug. 8, 1997; revised Oct. 20, 1997; accepted Oct. 23, 1997.

This work was supported by National Institute of Neurological Diseases and Stroke Grant PO1 NS19632, the James S. McDonnell Foundation, and the Centennial Medical Research Council (Canada). We are indebted to the valuable contribution of Jon Spradling for computerizing the delay tasks used in this study.

Correspondence should be addressed to Antoine Bechara, Department of Neurology, University of Iowa Hospitals and Clinics, Iowa City, IA 52242.

Copyright (C) 1997 Society for Neuroscience $0270-6474 / 97 / 180428-10 \$ 05.00 / 0$ et al., 1994). Using an experimental paradigm, the gambling task, which simulates real-life situations in the way it factors uncertainty, reward, and punishment (Bechara et al., 1994), VM subjects are unable to choose advantageously during the performance of this task, despite the correct knowledge of which are the good and bad decks in the task (Bechara et al., 1994; Bechara et al., 1996; Bechara et al., 1997). These studies suggest that the VM cortex is necessary for decision making. It is less clear whether the VM cortex is necessary for working memory, in view of the sensitivity of tasks used so far.

The primary objective of this investigation was to determine whether defects in decision making and working memory could be dissociated. To achieve this objective, we relied on the use of delayed task procedures to measure working memory, and on the gambling task to measure decision making. Several studies have established that the use of a variety of delay task procedures provides a valid measure of working memory (Goldman-Rakic, 1987, 1992; Jonides et al., 1993; Petrides et al., 1993; McCarthy et al., 1994; D'Esposito et al., 1995a; Smith et al., 1995; Swartz et al., 1995; Fuster, 1996). Other studies have established that the gambling task also provides a valid measure of decision making (Bechara et al., 1994; Bechara et al., 1996; Bechara et al., 1997). Our rationale for the idea that working memory and decision making are dissociable comes from (1) the observations that VM subjects suffer from impairments in decision making but preserve a normal level of memory and intellect (Eslinger and Damasio, 1985; Damasio et al., 1990; Damasio, 1996); on the other hand, although some DL subjects complain of memory impairments, they do not appear to suffer from impairments in decision making, as judged from their behavior in real life; and (2) the theoretical argument that working memory provides the mechanism by which representations of various options and scenarios are held on-line over a period (Fuster, 1990; Baddeley, 1992; 
Goldman-Rakic, 1992). This mechanism, however, does not explain how one of those representations gets selected for action. Therefore, it has been proposed that another mechanism marks these various options and scenarios, which are temporarily held in working memory, with positive or negative values, and then from among this array of available options the most advantageous option is selected for action (Damasio et al., 1991; Damasio, 1994; Damasio, 1996). This mechanism, which underlies the selection of good from bad options, is what we refer to as decision making (Bechara et al., 1994; Bechara et al., 1996; Bechara et al., 1997). Given the role of the DL cortex in working memory (Fuster, 1996), versus the VM cortex and its links with the limbic system and the processing of reward and punishment (Damasio, 1996), we hypothesized that VM prefrontal structures are necessary for decision making but not for working memory, whereas DL prefrontal structures are necessary for working memory but not for decision making. Thus, we predicted that VM subjects would show impaired decision making, as assessed with the gambling task, but normal working memory, as assessed with a variety of delay tasks. On the other hand, we predicted that subjects with DL lesions would show impaired working memory but normal decision making, as assessed with the same tasks. We tested these predictions in a population of neurological patients with bilateral focal damage in the VM prefrontal cortices and inpatients with damage in the right or left dorsolateral prefrontal cortices.

\section{MATERIALS AND METHODS}

Normal controls were recruited through local advertisement, and they were paid for their participation. Subjects with frontal lobe lesions $(n=$ 19) were selected from the patient registry of the University of Iowa Division of Behavioral Neurology and Cognitive Neuroscience. All frontal subjects had undergone basic neuropsychological and neuroanatomical characterization according to the standard protocols of the Benton Neuropsychology Laboratory (Tranel, 1996) and the Laboratory of Neuroimaging and Human Neuroanatomy (Damasio and Damasio, 1989; Damasio and Frank, 1992; Damasio, 1995).

All subjects provided informed consent. None of the subjects in this study had a history of mental retardation, learning disability, psychiatric disorder, substance abuse, or systemic disease that may affect the CNS.

The selection of subjects with brain lesions conformed to the following criteria: (1) a stable and chronic lesion (at least 3 months after onset), (2) bilateral involvement of orbital and ventromedial cortices, and (3) unilateral involvement of prefrontal and premotor cortices. Because subjects with lesions restricted to the DL prefrontal cortices are relatively rare, we decided to include also subjects with high mesial lesions. Thus, subjects with unilateral DL and/or high mesial lesions were included in a group that we will designate from here on as the $\mathrm{DL} / \mathrm{mesial}(\mathrm{DL} / \mathrm{M})$ group.

\section{Characteristics of the control and brain-damaged subject groups}

The control group included 14 women and 7 men $(n=21)$ with an age range from 24 to 68 years and 7-19 years of education. The braindamaged groups included nine VM subjects (three women and six men), four right DL/M subjects (all men), and six left DL/M subjects (one woman and five men). The subjects in all frontal lobe groups had an age range from 30 to 68 years and $8-18$ years of education. The neuropsychological profiles for subjects with brain damage are shown in Table 1.

\section{Anatomical analysis}

The description of extent of brain damage was done on the basis of lesion overlap among brain-damaged subjects in each group. All lesions of individual subjects were transferred onto a normal reference brain using the MAP-3 technique (Frank et al., 1997). In brief, the method entails the following: (1) a normal three-dimensional brain that is sliced in such a way that the slices match the slices of the magnetic resonance or computed tomographic scan of the subject with the brain lesion; a match between the slices of the two brains is thus created; (2) the contour of the lesion is transposed manually onto the slices of the normal brain, taking into consideration the relation of the lesion and the identified pertinent anatomical landmarks; and (3) for each lesion the set of contours constitutes an "object" that can be co-rendered with the normal brain. The objects corresponding to the different lesions in the group can intersect in space and thus can yield a maximal overlap relative to both surface and depth extension of damage. The number of subjects contributing to the overlap is known in each case.

\section{Characteristics of the experimental tasks}

Controls and brain-damaged subjects were tested on two sets of behavioral tasks: (1) the gambling task to test decision making, and (2) the delayed response and delayed nonmatching to sample tasks (with repeated stimuli) to test working memory.

The rationale for using two types of delay tasks was based on studies in nonhuman primates showing that different areas of the dorsolateral frontal cortex are associated with different domains of working memory: the inferior areas have been associated with object memory, whereas the superior areas have been associated with spatial memory (GoldmanRakic, 1987, 1992; Wilson et al., 1993). Similar dissociations were found recently in humans (Courtney et al., 1996). The delayed response tasks have been designed to tax the spatial (where) domain of working memory, whereas the delayed nonmatching to sample tasks are supposed to tax the object (what) domain of working memory (Fuster, 1990; Wilson et al., 1993). Because our DL/M lesions were not restricted to the inferior or superior regions, and the lesions spanned a wide area of DL cortices, we used both types of delayed tasks, because we anticipated that both domains of working memory (spatial and object) may be affected. In other words, we are not trying to sort out differences between different types of working memory but, rather, to cover a range of working memory with one task.

Testing of decision making. Decision making was assessed by a gambling task described in more detail previously (Bechara et al., 1994). In brief, this task entails the following.

Subjects sit in front of four decks of cards and are given a \$2000 loan of play money (a set of facsimile U.S. bills); the goal is to win as much money as possible. The subjects are told that the game requires a long series of card selections, one card at a time, from any of the four decks, until they are told to stop. After turning each card, the subject receives some money (the amount is only announced after the turning and varies with the deck). After turning some cards, the subject is both given money and asked to pay a penalty (again the amounts are only announced after the card is turned and vary with the deck and the position of the card in the deck according to a schedule unknown to the subjects). Turning any card from deck A or deck B yields $\$ 100$; turning any card from deck C or deck D yields $\$ 50$. However, the ultimate future yield of each deck varies, because the penalty amounts are higher in the high-paying decks (A and $\mathrm{B})$, leading to a negative balance, and lower in the low-paying decks (C and D), allowing a final gain. Thus, decks A and B are "disadvantageous," whereas decks C and D are "advantageous." Scores are the total numbers of cards selected from decks A and B (bad decks) versus the total numbers of cards selected from decks C and D (good decks).

Testing of working memory. Delay tasks that are used in nonhuman primates are too simple for use with humans. Therefore, we introduced a distractor during the delay between the cue and the response. The purpose of the distractor was to interfere with the ability of the subject to rehearse the position or the color of the cues during the delay and to increase the demands of the tasks on working memory.

\section{Delayed response experiment}

The subject sat in front of a computer screen on which four cards appeared for $2 \mathrm{sec}$. Two of the cards were face down, and the other two were face up showing red and/or black colors. The two face-up cards were randomly positioned among the four cards, and they also randomly changed from one trial to the next. The subjects were asked to pay attention to the four cards before they disappeared from the screen. Initially, the cards disappeared for one second and then reappeared, but this time all of the cards were face down. The subjects were told to select two of the four cards. The subjects were expected to discover, by themselves, that the two cards that were first face up were the correct ones to select. When a subject selected the two correct cards, a message appeared on the screen indicating that the subject had made the correct choice, and a $\$ 100$ bill was added to a pile of money appearing at the bottom left corner of the computer screen. If the subject selected one or two incorrect cards, the message indicated that the subject had made an incorrect choice, and $\$ 100$ was deducted from the money pile. 
Table 1. Demographic and neuropsychological data (underlined scores are defective)

\begin{tabular}{|c|c|c|c|c|c|c|c|c|c|c|c|c|c|c|c|}
\hline & \multirow[b]{2}{*}{ Gender } & \multirow[b]{2}{*}{ Age } & \multirow[b]{2}{*}{ Education } & \multirow[b]{2}{*}{$\mathrm{VIQ}^{a}$} & \multirow[b]{2}{*}{$\mathrm{PIQ}^{a}$} & \multirow[b]{2}{*}{$\mathrm{WMS} \mathrm{MQ}^{b}$} & \multicolumn{2}{|c|}{$\mathrm{BVRT}^{c}$} & \multirow{2}{*}{$\begin{array}{l}\text { Attn/ } \\
\text { Conc }^{d}\end{array}$} & \multirow[b]{2}{*}{ Speech } & \multirow[b]{2}{*}{$\mathrm{COWA}^{e}$} & \multicolumn{2}{|c|}{$\mathrm{WCST}^{f}$} & \multirow[b]{2}{*}{$\mathrm{FRT}^{g}$} & \multirow[b]{2}{*}{$\mathrm{JLO}^{h}$} \\
\hline & & & & & & & $\mathrm{C}$ & $\mathrm{E}$ & & & & Cat & PE & & \\
\hline EVR318 ${ }^{i}$ & M & 52 & 14 & 131 & 135 & 143 & 9 & 1 & 138 & Normal & 49 & 6 & 6 & 43 & 30 \\
\hline $\mathrm{PK} 0770^{i}$ & $\mathrm{~F}$ & 53 & 16 & 121 & 89 & 143 & 9 & 1 & 135 & Normal & 65 & 6 & 7 & $\underline{34}$ & 21 \\
\hline $\mathrm{KC} 1857^{i}$ & M & 30 & 14 & 96 & $\overline{81}$ & $92 \mathrm{r}$ & 6 & 7 & 106 & Normal & 38 & 6 & 8 & $\overline{43}$ & 23 \\
\hline DV1589 ${ }^{i}$ & M & 45 & 18 & 126 & 98 & $100 \mathrm{r}$ & 7 & 4 & 112 & Normal & 41 & 6 & 5 & 43 & 23 \\
\hline VY0500 & $\mathrm{F}$ & 64 & 12 & 113 & 88 & $\underline{90 \mathrm{r}}$ & 6 & 6 & 109 & Normal & 31 & 4 & $\underline{36}$ & 45 & 29 \\
\hline $\mathrm{TK} 1255^{j}$ & M & 47 & 12 & 87 & 86 & 112 & 6 & 6 & & Normal & 40 & 5 & 20 & 47 & 25 \\
\hline $\mathrm{JL} 1445^{j}$ & M & 51 & 9 & 91 & 86 & 79 & $\underline{4}$ & $\underline{12}$ & 95 & Normal & 39 & 6 & 32 & $\underline{32}$ & \\
\hline $\mathrm{RS} 1479^{j}$ & $\mathrm{~F}$ & 63 & 8 & 87 & 88 & $95 r$ & 5 & 7 & 75 & Normal & 51 & $\underline{0}$ & $\underline{84}$ & 44 & 22 \\
\hline $\mathrm{JR} 1584^{j}$ & M & 51 & 8 & 90 & 111 & $\underline{67 \mathrm{r}}$ & 7 & 6 & 108 & Normal & 25 & 6 & 10 & $\underline{39}$ & 30 \\
\hline WG0827 ${ }^{k}$ & M & 68 & 12 & $\overline{92}$ & 85 & $\overline{129}$ & 9 & 1 & & Normal & 21 & & & 42 & 29 \\
\hline $\mathrm{AH} 1331^{k}$ & M & 63 & 12 & 117 & 95 & 132 & 7 & 4 & & Normal & 45 & 6 & 18 & 50 & 23 \\
\hline IM1725 & M & 66 & 12 & 94 & 85 & & 5 & $\underline{11}$ & & Normal & 32 & & & $\underline{39}$ & $\underline{14}$ \\
\hline $\mathrm{RB} 1561^{k}$ & M & 56 & 16 & 113 & 129 & & 7 & 5 & & Normal & 39 & 6 & 8 & $\overline{43}$ & 29 \\
\hline DM0414 ${ }^{l}$ & M & 48 & 12 & 87 & 87 & 103 & $\underline{4}$ & $\underline{11}$ & 83 & Normal & $\underline{20}$ & 5 & $\underline{28}$ & 50 & 22 \\
\hline $\mathrm{JG} 0468^{l}$ & M & 68 & 16 & 111 & 132 & 112 & 9 & 1 & 97 & Normal & $\overline{44}$ & 6 & 13 & 50 & 30 \\
\hline RS0868 & $\mathrm{F}$ & 75 & 14 & 74 & 97 & & 8 & 5 & & Broca & $\underline{12}$ & & & 49 & 30 \\
\hline $\mathrm{JD} 1195^{l}$ & M & 65 & 15 & 123 & 107 & 123 & 8 & 3 & 107 & Normal & 40 & $\underline{3}$ & $\underline{29}$ & 42 & 30 \\
\hline $\mathrm{RD} 1649^{l}$ & M & 73 & 16 & 118 & 144 & $104 \mathrm{r}$ & 6 & 6 & 118 & Normal & 38 & $\underline{1}$ & $\underline{40}$ & 47 & 30 \\
\hline HL1812 & M & 43 & 16 & 113 & 118 & & 6 & 4 & & $\mathrm{WFD}^{m}$ & $\underline{16}$ & 6 & 8 & $\underline{39}$ & \\
\hline
\end{tabular}

${ }^{a}$ VIQ, Verbal IQ; PIQ, performance IQ (from the WAIS-R).

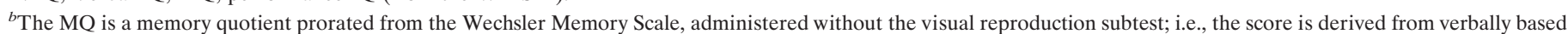
subtests only. MQs accompanied by an $\mathrm{r}$ are the verbal index from the Wechsler Memory Scale-Revised.

${ }^{c}$ BVRT, Benton Visual Retention Test; C, number correct (\#/10); E, number of errors.

${ }^{d}$ Attn/Conc, Attention/concentration index from the Wechsler Memory Scale-Revised.

${ }^{e}$ COWA, Controlled Oral Word Association Test from the Multilingual Aphasia Exam.

${ }^{f}$ WCST, Wisconsin Card Sorting Test; Cat, categories completed; PE, perseverative errors.

${ }^{g}$ FRT, Facial Recognition Test.

${ }^{h}$ JLO, Judgment of Line Orientation Test.

${ }^{i}$ Abnormal gambling/normal delay.

${ }^{j}$ Abnormal gambling/abnormal delay.

${ }^{k}$ Right frontal lesions.

${ }^{l}$ Left frontal lesions.

${ }^{m}$ WFD, Word-finding defect.

After discovering the rules and reaching a learning criterion of five consecutive correct choices, the time delay between the appearance and reappearance of the cards began to alternate between 10,30 , or $60 \mathrm{sec}$ in a random manner. During the delay, the subject had to read aloud a series of semantically meaningless sentences. Subjects were told that the goal in this game was to win as much money as possible. The task consisted of completing 15 trials in each of the 10, 30, and $60 \mathrm{sec}$ delay categories. Scores were calculated as the percent correct choices made by the subject at the 10, 30, and $60 \mathrm{sec}$ delays. Impaired performance on the delayed response task was defined as achieving a correct score of $\leq 80 \%$ at the 60 sec delay, a cutoff score below which no normal control ever performed (see Results).

\section{Delayed nonmatching to sample experiment}

In this experiment, the task was similar to the delayed response task, except that only one card appeared initially on the computer screen for 2 sec. The card was face up and was either red or black. The red or black color of the card randomly changed from one trial to the next. The card disappeared for one second, and then four cards appeared on the screen. All the cards were face up; two of them were red, and two were black. The positions of the red and black cards were random. The subjects were expected to discover, by themselves, that selecting the two cards that were opposite in color (nonmatching) to the initial sample card was the correct response. After discovering the rules and reaching a learning criterion of five consecutive correct responses, the time delay between the appearance of the sample card and the appearance of the two matching and two nonmatching cards began to alternate among 10,30 , or $60 \mathrm{sec}$ in a random manner. During the delay, the subject was distracted as in the previous experiment. The task consisted of completing 15 trials in each of the 10, 30, and $60 \mathrm{sec}$ delay categories. Scores were calculated as the percent correct choices made by the subject at the 10,30 , and 60 sec delays. Impaired performance on the delayed nonmatching to sample task was defined as achieving a correct score of $\leq 80 \%$ at the $60 \mathrm{sec}$ delay, a cutoff score below which no normal control ever performed (see Results).

\section{RESULTS}

\section{Lesion description in brain-damaged groups}

The overlap maps of lesions for the three groups of braindamaged subjects are shown in Figure 1. The lesions in the VM group (Fig. 1 $A$ ) showed a maximum overlap in the ventral and low mesial sectors of the frontal lobe, in both the right and left hemispheres. Some of the lesions also extended to involve the frontopolar region and the most anterior sectors of the dorsolateral regions. The DL/M lesions (six on the left and four on the right) (Fig. $1 B$ ) covered the dorsolateral sector of the frontal lobes but did not reach the polar region. They also involved the high medial sectors of the frontal lobes above the level of the body of the corpus callosum. They did not reach the low mesial sector below this level of the callosum. In the right hemisphere, the lesions also extended into the orbital sector but stayed in the lateral half, not involving the mesial orbital sector. 
Figure 1. Overlap of lesions in the three groups of brain-damaged subjects. $A$, Bilateral VM lesions. $B$, Right and left DL/M lesions. $C$, Color bar showing the color code corresponding to number of overlap of lesions.

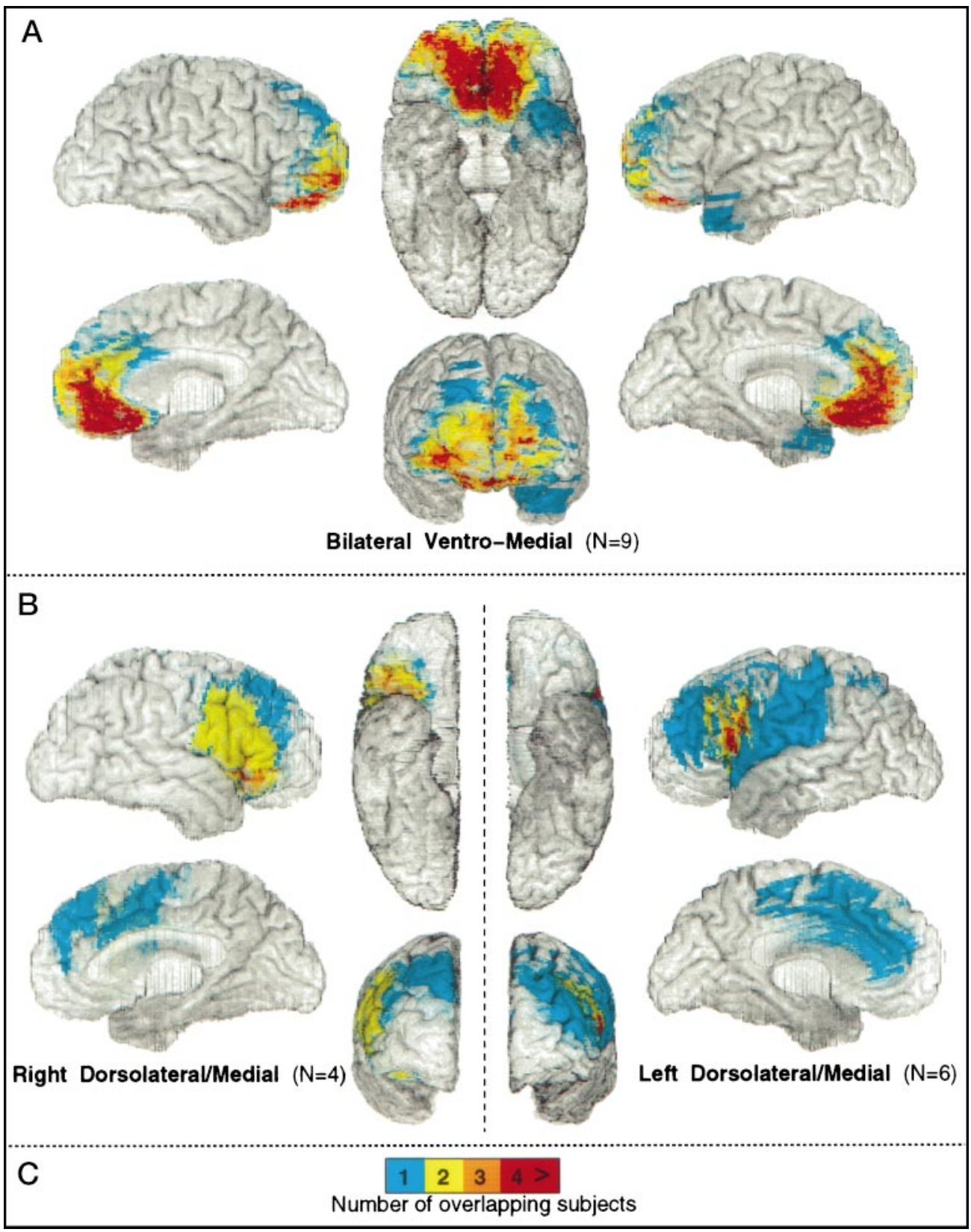

B

Right DorsolateralMedial $(\mathrm{N}=4)$

Left Dorsolateral/Medial $(\mathrm{N}=6)$

\section{Task results in normal controls}

Normal controls selected an average of 62 cards from the good decks versus 38 cards from the bad decks. Based on unpublished normative data, we set a cutoff point at which the selection of $>50$ cards from the bad decks (i.e., $<50$ cards from the good decks) was classified as defective.

Normal controls achieved a high percent correct score on both the delayed response and delayed nonmatching to sample tasks. In our comparisons (below), we used the percent correct responses obtained at the $60 \mathrm{sec}$ delay as our dependent variable, because in our task, this is the time when demand for working memory was the highest. The performance of normal controls at the $60 \mathrm{sec}$ delay was $\sim 95 \%$ correct for the delayed response task and $90 \%$ correct for the delayed nonmatching to sample task. No normal control achieved a score of $<80 \%$ correct. Thus scores of $<80 \%$ were classified as defective.

\section{Task results in brain-damaged subjects Gambling task}

Nine out of nine subjects in the VM group selected $>50$ cards from the bad decks; i.e., all were impaired on the gambling task (Fig. 2).

Ten of 10 subjects in the the DL/M group selected $>50$ cards from the good decks; i.e., all were normal on the gambling task. When the gambling task scores from right DL/M subjects and the scores from left DL/M subjects were analyzed separately, there were no statistical differences in the number of cards selected from the good or bad decks by right DL/M subjects compared with normals (Mann-Whitney $U$ test $Z=-0.92 ; p>0.1$ ) or by left DL/M subjects compared with normals (Mann-Whitney $U$ test $Z=-0.94 ; p>0.1)$. Nonetheless, there was some difference in the level of the normal performance of the right DL/M group relative to the left. Although the average performance of the left 


\section{Decision Making}

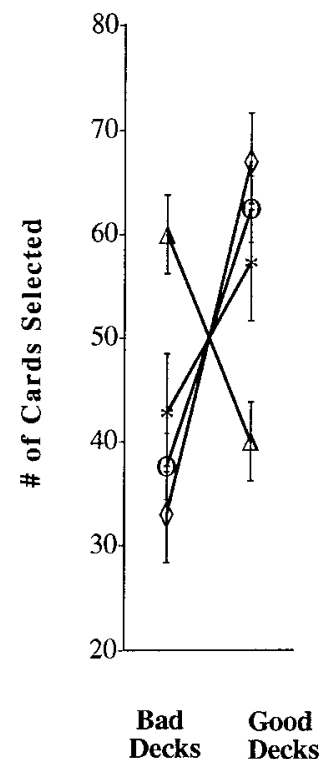

Delayed

Response

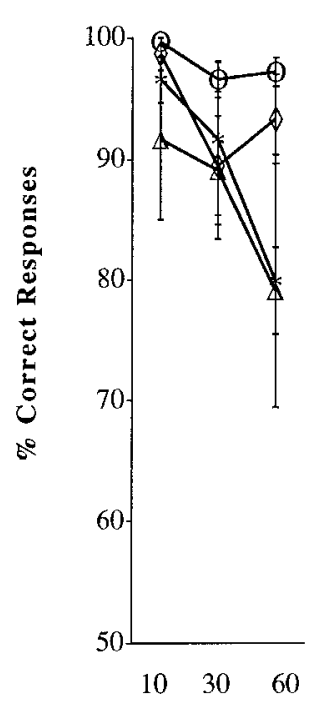

Delay in Seconds

\section{Delayed Non-Matching to Sample}

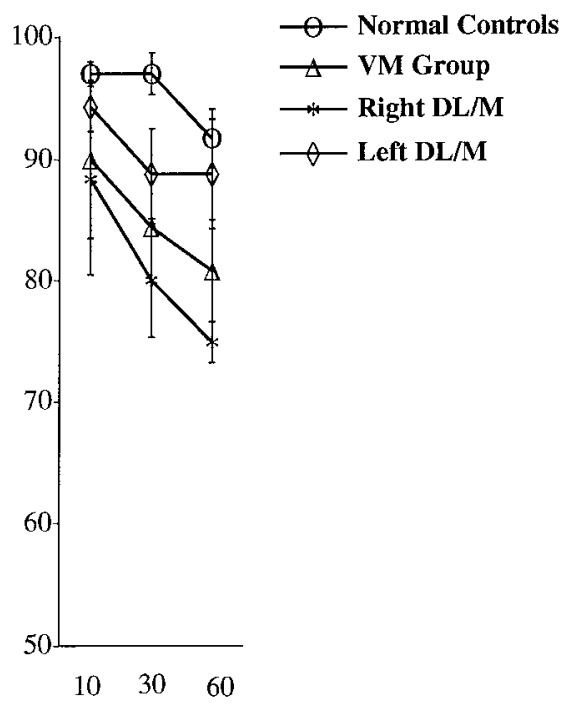

Delay in Seconds

Figure 2. Comparison of the performance of the three brain-damaged groups and the normals. Each graph represents mean \pm SEM of the percent correct responses, or total number of cards selected from the good versus the bad decks, that were made by normal controls $(n=21)$, by subjects with bilateral orbital and VM frontal lobe lesions $(n=9)$, by subjects with lesions in the right DL/M sector of the prefrontal cortex $(n=4)$, or by subjects with lesions in the left DL/M sector of the prefrontal cortex $(n=6)$. We note that every participant in the delayed response and delayed nonmatching to sample tasks reached a $100 \%$ learning criterion at the $0 \mathrm{sec}$ delay before the task could proceed to the 10,30 , or $60 \mathrm{sec}$ delays.

DL/M subjects was just as good, or even better, than that of normal controls, the average performance of right DL/M subjects was in the low normal range (Fig. 2).

\section{Delay tasks}

As a group, subjects with VM lesions were mildly impaired on the delayed response task and were borderline on the delayed nonmatching to sample task (Fig. 2). However, it was interesting to see that the performance on these tasks was not uniformly abnormal or borderline. In fact, the group could be subdivided into two subgroups, in which five of the VM subjects had impaired performance on both delay tasks, whereas four of the subjects had normal performance on both delay tasks.

In the four subjects with VM lesions and normal delay performance, the scores were indistinguishable from controls, and if anything, their scores were even better than normal controls (Fig. 3). In the other five subjects (VM lesions and abnormal delay task performance), the degrees of impairment were similar on both delay tasks (Fig. 3). At the $60 \mathrm{sec}$ delay, these subjects had significantly lower scores than normal controls (Mann-Whitney $U$ tests revealed $Z=-3.11 ; p<0.01$ for delayed response, and $Z=$ $-3.06 ; p<0.01$ for delayed nonmatching to sample). Because Figure 3 indicates that the degree of impairment increased as a function of the length of the delay, the results suggest that the impairment was related to memory.

In the DL/M group, three subjects with right hemisphere lesions showed abnormal performance on both delay tasks. One subject (with a lesion restricted to the inferior area of the dorsolateral sector on the right) showed selective impairment on the delayed nonmatching to sample but not on the delayed response task. The six subjects with left hemisphere lesions had normal performances on both delay tasks (Fig. 2).
The degree of impairment on the delayed response task in the right $\mathrm{DL} / \mathrm{M}$ subjects was less severe than that of the delayed nonmatching to sample because of the one subject who did not show a deficit on the delayed response task. At the $60 \mathrm{sec}$ delay, these subjects achieved significantly lower scores than normal controls (Mann-Whitney $U$ tests revealed $Z=-2.2 ; p<0.05$ for delayed response, and $Z=-2.8 ; p<0.05$ for delayed nonmatching to sample). Because the figure indicates that the degree of impairment appeared to increase as the time of delay got longer, the results suggest a memory-related impairment. The finding of normal performance on the delayed response task and abnormal performance on the delayed nonmatching to sample task in the one subject whose lesion was restricted to the inferior sector of the dorsolateral sector is consistent with several previous studies (Goldman-Rakic, 1987, 1992; Wilson et al., 1993; Courtney et al., 1996).

The left DL/M subjects appeared to have lower scores than normals at the 30 and $60 \mathrm{sec}$ delays, but these differences were not statistically significant.

\section{Further anatomical investigation of the VM subjects}

Because of our finding of a split in the delay task performance in the VM group, five subjects with abnormal delay task performance and four subjects with normal performance, we decided to analyze these two subgroups separately: group 1, those with both abnormal gambling task performance and abnormal delay task performance; and group 2, those with abnormal gambling task performance but normal delay task performance (Fig. 3).

This split in performance of VM subjects on the delay tasks but not on the gambling task was intriguing. We suspected an anatomical reason underlying this separation. Therefore, we looked at the overlap of lesions in these two groups separately. In group 


\section{Decision Making}

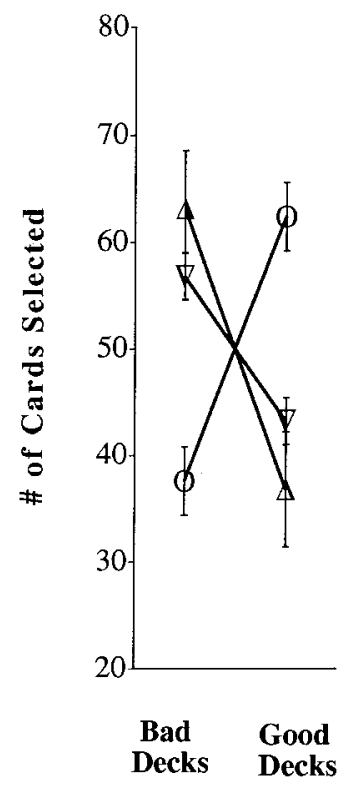

Delayed Response

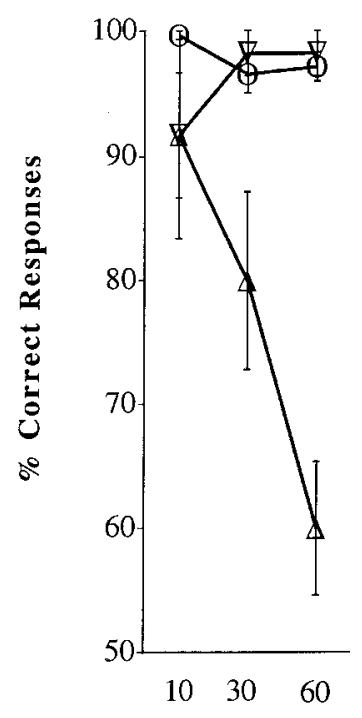

Delay in Seconds Delayed Non-Matching to
Sample

Figure 3. Mean \pm SEM of the percent correct responses, or total number of cards selected from the good versus the bad decks, that were made by normal controls $(n=21)$ and by subjects with bilateral orbital and VM frontal lobe lesions who were divided into two groups based on their performance on the delayed response and delayed nonmatching to sample tasks: group 1 (abnormal gambling and abnormal delay) $(n=5)$ and group 2 (abnormal gambling and normal delay) $(n=4)$.

1 (abnormal gambling and abnormal delay), all subjects had lesions that extended posteriorly, possibly involving the basal forebrain region. However, in group 2 (abnormal gambling and normal delay), the lesions were more anterior and probably did not involve the basal forebrain region (Fig. 4). We purposefully state that the lesions "probably" involved (or did not involve) the basal forebrain region; because of the nature of many of these lesions, their surgical treatment (clipping of ruptured aneurysms), and the unavoidable artifact they induce, a clear-cut decision of "yes" or "no" involvement is not possible.

\section{Further statistical analyses}

When the gambling task scores from subjects classified as group 1 and the gambling scores from subjects classified as group 2 were analyzed separately, there was no difference in the severity of impairment on the gambling task. We conducted a two-way ANOVA comparing the number of cards selected by normal controls and group 1 and group 2 subjects (a between-group comparison). The comparison was made with regard to the number of cards selected from the good decks versus the bad decks (a within-group comparison). The analysis revealed a significant interaction of group with decks $\left(F_{(2,15)}=13.3 ; p<0.001\right)$, reflecting the fact that both $\mathrm{VM}$ groups selected more cards from the bad decks, whereas the controls selected more cards from the good decks. Post hoc Newman-Kuels tests revealed that the numbers of cards selected from the good decks by normal controls were signifcantly higher than those selected from the bad decks. By contrast, the numbers of cards selected from the bad decks by both VM groups were significantly higher than those selected from the good decks $(p<0.01)$. It is worth mentioning that the performance of group 1 (abnormal gambling and abnormal delay) subjects appeared worse than that of group 2 subjects (i.e., they selected more bad cards and less good cards). However, this difference was not statistically significant (Fig. 3).

The group 2 subjects had abnormal gambling (i.e., selected fewer cards from the good decks) but normal delay task scores. By contrast, the right DL/M subjects had normal gambling but abnormal delay task scores (Fig. 5). To confirm statistically the reliability of the finding of a double dissociation in the deficits associated with anterior VM and right DL/M lesions, we conducted the following analysis. The scores from the two delay tasks at the $60 \mathrm{sec}$ delay were merged into a single score by taking an average of the two. The two scores reflecting the number of cards from the good or bad decks on the gambling task were also merged into a single score equal to the number of cards from the good decks minus the number of cards from the bad decks. Thus, a positive number reflects an advantageous performance, whereas a negative number reflects a disadvantageous performance. A two-way ANOVA comparing the group 2 (anterior VM) and right $\mathrm{DL} / \mathrm{M}$ groups (between-group comparison) on the scores from the delay versus the gambling tasks (within-group comparison) revealed a significant interaction of groups with tasks $\left(F_{(1,6)}\right.$ $=35.0 ; p<0.001$ ), reflecting the fact that the group 2 (anterior VM) subjects had high delay task scores (i.e., normal performance) and negative gambling scores (i.e., abnormal performance), whereas the right $\mathrm{DL} / \mathrm{M}$ subjects had low delay task scores (abnormal) and positive gambling task scores (normal). Post hoc Newman-Kuels tests confirmed that the delay task scores from group 2 (anterior VM) were significantly higher than those from right $\mathrm{DL} / \mathrm{M}$, whereas the gambling scores were signifcantly lower $(p<0.01)$. 


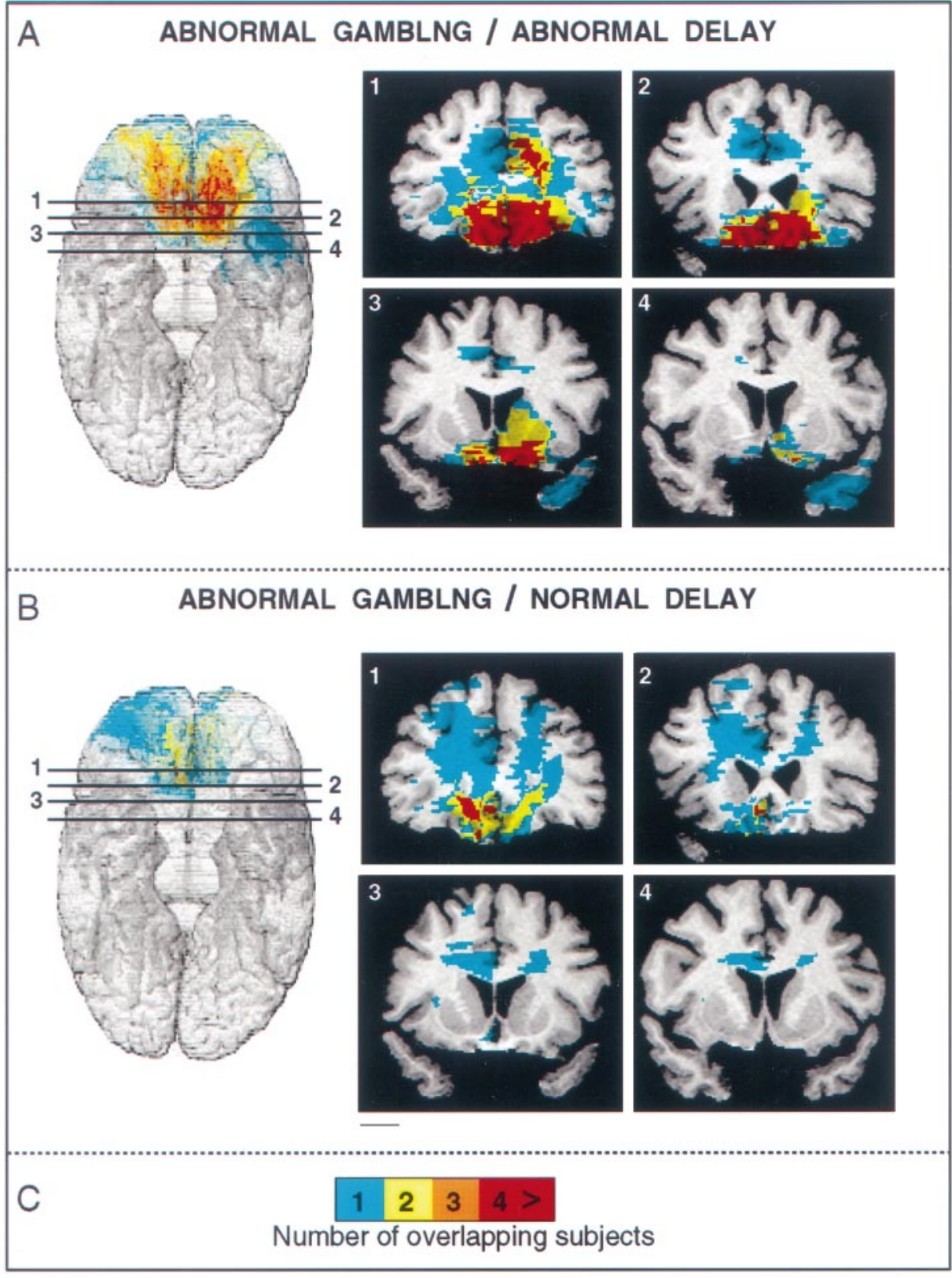

Figure 4. Separate mapping of VM lesions for group $1(A)$ and group $2(B)$ subjects. The maximal overlap of subjects in $A$ is seen spanning the whole extent of the mesial orbital surface of the frontal lobe. It reaches the most posterior sector (coronal slices 3,4) where basal forebrain structures are found. However, in $B$ the maximal overlap is mostly anterior, extending only to slices 1 and 2 . Slices 3 and 4 do not show any lesion. Coronal sections are arranged according to radiological convention, i.e., right is left, and vice versa.

\section{DISCUSSION}

Our initial hypothesis was that the VM prefrontal structures would be necessary for decision making but not for working memory, whereas the DL prefrontal structures would be necessary for working memory but not for decision making. Our results show that, in fact, all subjects with VM lesions are impaired on the gambling task, whereas only a subset of these subjects, those with the most anteriorly placed lesions, presumably sparing the basal forebrain structures, are normal on the delay tasks. As predicted, subjects with right DL/M lesions were impaired on the delay tasks but not on the gambling task. These results suggest a double dissociation between impairments in these two tasks, that is, between decision making on the one hand and working memory for the spatial and object domains on the other.

Our initial prediction that we would find a complete double dissociation between decision making and working memory relative to the VM and DL sectors of the prefrontal cortex, however, has to be revised. The results suggest that a working memory impairment influences, to some extent, decision making, given that (1) the subjects in the right $\mathrm{DL} / \mathrm{M}$ group performed at a low normal level in the gambling task; and that (2) the VM subjects with posterior lesions and abnormal performance on the delay tasks showed the worst performance on the gambling task. We interpret these findings as evidence that working memory and decision making may be asymmetrically dependent. Working memory is not dependent on the intactness of decision making; i.e., subjects can have normal working memory in the presence or absence of deficits in decision making. On the other hand, decision making seems to be influenced by the intactness or impairment of working memory; i.e., the subject's decision making is affected by having an abnormal working memory.

Subjects with left DL/M lesions were normal on both the gambling and delay tasks. The normal gambling task performance had been predicted, and the absence of a working memory im- 


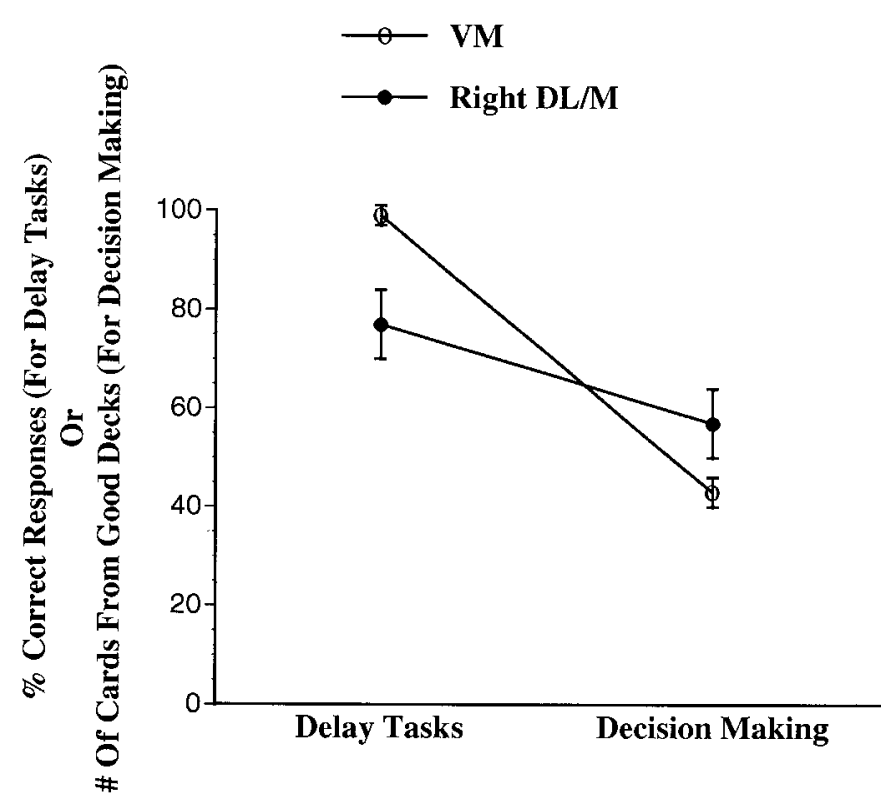

Figure 5. Mean \pm SEM of the average of percent correct responses from the two delay tasks, or the total number of cards selected from the good decks, that were made by VM subjects with more anterior lesions (group 2 ), and by subjects with right DL/M lesions.

pairment is not surprising, because, during the delay, the verbal memorization of cues was probably avoided by the interference procedure, thus rendering the task primarily nonverbal. This is consistent with several functional neuroimaging studies in humans that showed higher activation in the right dorsolateral frontal cortex, relative to the left, during the performance of similar delay tasks (Jonides et al., 1993; Petrides et al., 1993; McCarthy et al., 1994; D’Esposito et al., 1995a,b; Smith et al., 1995; Swartz et al., 1995).

It could be argued that the reason for the separation of the two VM groups is related to the size of the lesion, rather than to the placement of the lesion. This, however, is not the case. As can be seen in Figure 6, EVR318, who has a large bilateral frontal lesion, has abnormal performance in the gambling task but normal performance in the delay task. On the other hand, VY500 has a smaller, more limited lesion in the posterior orbital and mesial sector of the frontal lobe and is impaired on both tasks. A large dorsolateral lesion in the right frontal lobe, as in case AH1331, which even involves the lateral part of the orbital sector but spares its medial portion, has normal performance in the gambling task, whereas the delayed response is abnormal. Yet DV1589, with a small lesion in the mesial orbital sector, mostly on the right, shy of the basal forebrain region and much smaller than the lesion of AH1331, does show a deficit in the gambling task but not the delay task. In sum, it is the placement of the lesion, rather than its size, that accounts for the observed deficits.

We draw support for our conclusion that subjects with right $\mathrm{DL} / \mathrm{M}$ lesions have a working memory deficit in spatial and object domains from the evidence of a large body of studies demonstrating that structures within the dorsolateral prefrontal cortices are implicated in working memory (Goldman-Rakic, 1987, 1992; Jonides et al., 1993; Petrides et al., 1993; McCarthy et al., 1994; D'Esposito et al., 1995a; Smith et al., 1995; Swartz et al., 1995; Fuster, 1996). We also conclude that subjects with posterior VM lesions involving the basal forebrain region might have had abnormal performance on the delay tasks because of an impairment
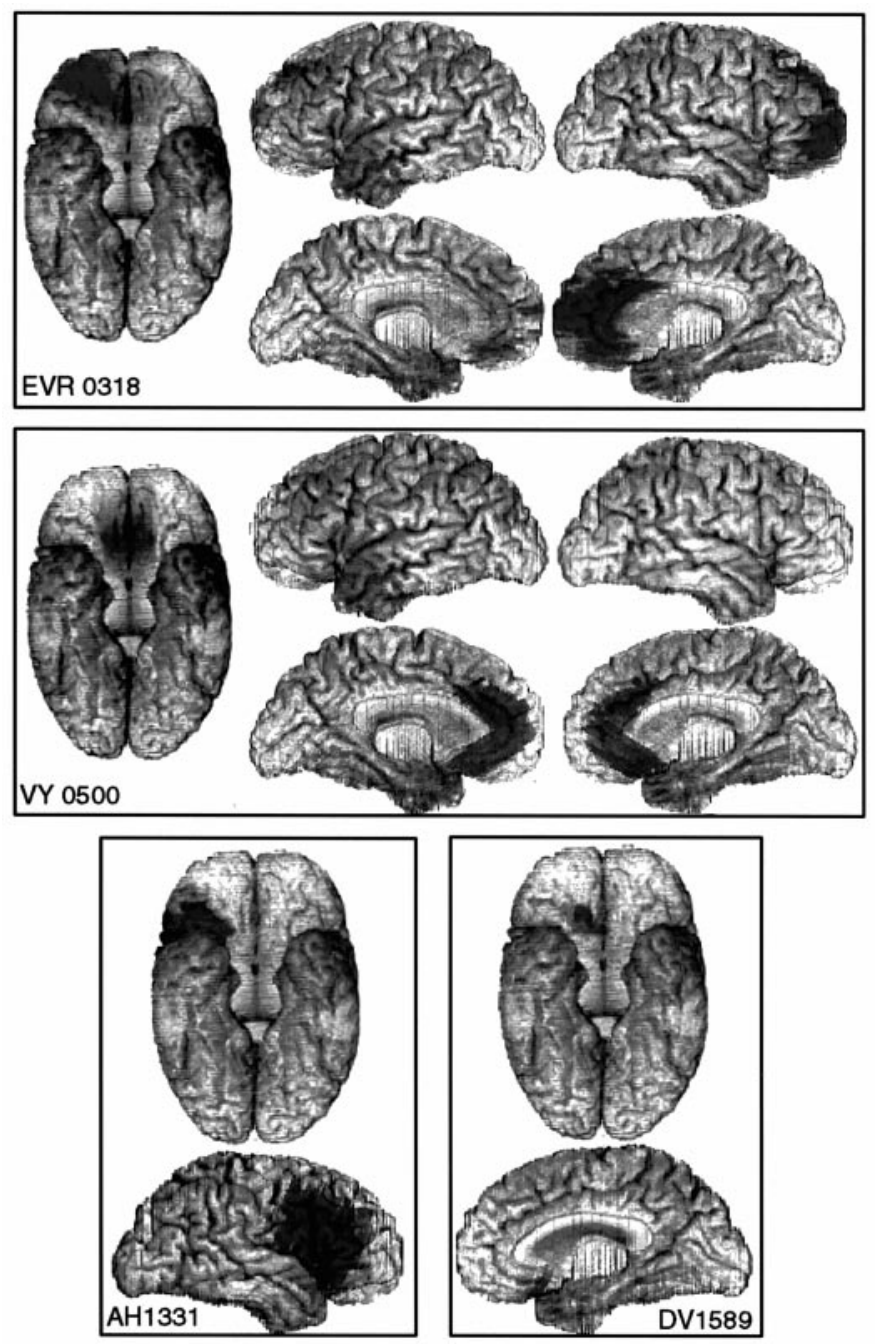

Figure 6. Examples of mapped individual cases from the VM and DL/M groups demonstrating that placement of the lesion, rather than the size of the lesion, is the crucial variable in producing the various combinations of deficits (see Discussion for detail).

of working memory in those domains. That such subjects have memory defects had been established previously (Damasio et al., 1985; Markowitsh and Pritzel, 1985), and it seems plausible that the basal forebrain region may serve as a parallel system to the dorsolateral prefrontal cortex for working memory (Swartz et al., 1995), given the close anatomical links between the two regions (Devito and Smith, 1964). In the posterior VM group, the memory deficit might have had an additional effect on their decision making capacity and contributed to the fact that, as a group, these subjects had the worst performance in the gambling task.

Our findings of a separation of VM lesions into two distinct groups is important and may explain the equivocal results of earlier lesion studies in humans on the effects of VM damage on the performance of delay tasks (Ghent et al., 1962; Chorover and Cole, 1966; Freedman, 1986). It is likely that in these earlier studies some lesions may have extended posteriorly and involved the basal forebrain region, whereas others did not, thus resulting in a mixed group. Our results are also compatible with findings of functional neuroimaging studies in humans that did not reveal any activation within the anterior orbital and ventromedial prefrontal cortices during the performance of a variety of delay task 
procedures (Jonides et al., 1993; Petrides et al., 1993; McCarthy et al., 1994; D'Esposito et al., 1995a; Smith et al., 1995; Cohen et al., 1997; Courtney et al., 1997). In the only case in which ventromedial frontal activation was observed, it was found in the region closest to the basal forebrain (Swartz et al., 1995), a finding that again is in agreement with our results presented here.

Still, other possible explanations for the defective performance on the gambling task, which were not specifically tested in this study, include impairments in response inhibition and selective attention. Indeed, the VM prefrontal cortex is important for response inhibition and attentional shifting in both animals (Mishkin, 1964; Fuster, 1991; Dias et al., 1996) and humans (Diamond, 1990; Stuss et al., 1992). Furthermore, in addition to impairments in working memory, studies in nonhuman primates have shown that impaired delay task performance can also result from impairments in selective attention (Heilman et al., 1987; Rizzolatti and Camarda, 1987) and response inhibition (Mishkin, 1964; Fuster, 1991; Dias et al., 1996; Fuster, 1996). Because in our gambling task the subjects were rewarded repeatedly before encountering a loss when choosing cards from a bad deck, it might be argued that the impaired performance of the VM subjects was caused by defective response inhibition, i.e., the inability to suppress previously rewarded responses and shifting attention to the good decks. However, this explanation is inconsistent with several facts. (1) VM subjects with lesions restricted to the anterior sector (abnormal gambling and normal delay) do not show any perseverative behavior and attentional deficit on conventional neuropsychological tests (Table 1). (2) When we analyze the profile of performance of these anterior VM subjects on the gambling task, we find that these subjects switch decks whenever they receive punishment, just as normal controls do, although they return more often to the decks that yield high immediate reward (the bad decks). The switch away from the bad deck immediately after a punishment does not indicate lack of inhibition of the natural tendency to shift decks after a negative outcome (Bechara et al., 1994). (3) Anterior VM subjects are not impaired on the delay tasks, which have been considered sensitive to deficits in selective attention and response inhibition as mentioned above. In short, we believe that these facts strongly argue against impairments in response inhibition and selective attention as an explanation for the impaired gambling task performance. We may even go as far as to conclude that the ability to select an advantageous response from among an array of response options is probably distinct from working memory, from response inhibition, and from selective attention.

It is clear that the failure of VM subjects to choose advantageously does not result from their failure to appreciate the value of each deck, because VM subjects continue to choose disadvantageously, even when they know which decks are good and which ones are bad (Bechara et al., 1997). We considered three possibilities for why VM subjects continue to prefer the bad decks over the good decks: (1) hypersensitivity to reward, in which the prospect of a large immediate gain outweighs any prospect of future loss; (2) insensitivity to punishment, in which the prospect of a large loss cannot override any prospect of gain; and (3) insensitivity to future consequences, positive or negative, in which the immediate prospects override any future prospects. Preliminary evidence (Anderson et al., 1996) suggests that in most VM subjects, the decision-making impairment is linked to insensitivity to future consequences, whatever they may be. Thus, the VM subject appears oblivious to the future and guided by only immediate prospects, positive or negative.

\section{REFERENCES}

Anderson SW, Damasio H, Jones RD, Tranel D (1991) Wisconsin card sorting test performance as a measure of frontal lobe damage. J Clin Exp Neuropsychol 3:909-922.

Anderson SW, Bechara A, Tranel D, Damasio H, Damasio AR (1996) Characterization of the decision-making defect of subjects with ventromedial frontal lobe damage. Soc Neurosci Abstr 22:711.

Baddeley A (1992) Working memory. Science 255:556-559.

Bechara A, Damasio AR, Damasio H, Anderson SW (1994) Insensitivity to future consequences following damage to human prefrontal cortex. Cognition 50:7-15.

Bechara A, Tranel D, Damasio H, Damasio AR (1996) Failure to respond autonomically to anticipated future outcomes following damage to prefrontal cortex. Cereb Cortex 6:215-225.

Bechara A, Damasio H, Tranel D, Damasio AR (1997) Deciding advantageously before knowing the advantageous strategy. Science 275:1293-1295.

Chorover S, Cole M (1966) Delayed alternation performance in patients with cerebral lesions. Neuropsychologia 4:1-7.

Cohen JD, Peristein WM, Braver TS, Nystrom LE, Noll DC, Jonides J, Smith EE (1997) Temporal dynamics of brain activation during a working memory task. Nature 386:604-608.

Courtney SM, Ungerleider LG, Keil K, Haxby JV (1996) Object and spatial visual working memory activate separate neural systems in human cortex. Cereb Cortex 6:39-49.

Courtney SM, Ungerleider LG, Keil K, Haxby JV (1997) Transient and sustained activity in a distributed neural system for human working memory. Nature 386:608-611.

D'Esposito M, Detre JA, Alsop DC, Shin RK, Atlas S, Grossman M (1995a) The neural basis of central execution systems of working memory. Nature 378:279-281.

D'Esposito M, Shin RK, Detre JA, Incledon S, Annis D, Aguirre GK, Grossman M, Alsop DC (1995b) Object and spatial working memory activates dorsolateral prefrontal cortex: a functional MRI study. Soc Neurosci Abstr 21:1498.

Damasio AR (1994) Descartes' error: emotion, reason, and the human brain. New York: Grosset/Putnam.

Damasio AR (1996) The somatic marker hypothesis and the possible functions of the prefrontal cortex. Philos Trans R Soc Lond [Biol] 351:1413-1420.

Damasio AR, Eslinger PJ, Damasio H, Van Hoesen GW, Cornell S (1985) Multimodal amnesic syndrome following bilateral temporal and basal forebrain damage. Arch Neurol 42:252-259.

Damasio AR, Tranel D, Damasio H (1990) Individuals with sociopathic behavior caused by frontal damage fail to respond autonomically to social stimuli. Behav Brain Res 41:81-94.

Damasio AR, Tranel D, Damasio H (1991) Somatic markers and the guidance of behavior: Theory and preliminary testing. In: Frontal lobe function and dysfunction (Levin HS, Eisenberg HM, Benton AL, eds), 217-229. New York: Oxford UP.

Damasio H (1995) Human brain anatomy in computerized images. New York: Oxford UP.

Damasio H, Damasio AR (1989) Lesion analysis in neuropsychology. New York: Oxford UP.

Damasio H, Frank R (1992) Three-dimensional in vivo mapping of brain lesions in humans. Arch Neurol 49:137-143.

Devito JL, Smith OA (1964) Subcortical projections of the prefrontal lobe of the monkey. J Comp Neurol 123:413-423.

Diamond A (1990) Developmental time course in human infants and infant monkeys, and the neural bases of, inhibitory control in reaching. In: The development and neural bases of higher cognitive functions (Diamond A, ed), pp 637-669. New York: New York Academy of Sciences.

Dias R, Robbins TW, Roberts AC (1996) Dissociation in prefrontal cortex of affective and attentional shifts. Nature 380:69-72.

Eslinger P, Damasio AR (1984) Behavioral disturbances associated with rupture of anterior communicating artery aneurysms. Semin Neurol 4:385-389.

Eslinger PJ, Damasio AR (1985) Severe disturbance of higher cognition after bilateral frontal lobe ablation: patient EVR. Neurology 35:1731-1741.

Frank R, Damasio H, Grabowski TJ (1997) Brainvox: an interactive multimodal visualization and analysis system for neuroanatomical imaging. NeuroImage 5:13-30. 
Freedman M (1986) Bilateral frontal lobe disease and selective delayed response deficits in humans. Behav Neurosci 100:337-342.

Fuster JM (1990) Prefrontal cortex and the bridging of temporal gaps in the perception-action cycle. In: The development and neural bases of higher cognitive functions (Diamond A, ed), pp 318-336. New York: New York Academy of Sciences.

Fuster JM (1991) The prefrontal cortex and its relation to behavior. In: Progress in brain research (Holstege G, ed), pp 201-211. New York: Elsevier.

Fuster JM (1996) The prefrontal cortex. Anatomy, physiology, and neuropsychology of the frontal lobe. New York: Raven.

Ghent L, Mishkin M, Teuber HL (1962) Short-term memory after frontal lobe injury in man. J Comp Physiol Psychol 55:705-709.

Goldman-Rakic PS (1987) Circuitry of primate prefrontal cortex and regulation of behavior by representational memory. In: Handbook of physiology; the nervous system (Plum F, ed), pp 373-401. Bethesda, MD: American Physiological Society.

Goldman-Rakic PS (1992) Working memory and the mind. Sci Am 267:111-117.

Grafman J, Jonas B, Salazar A (1990) Wisconsin Card Sorting Test performance based on location and size of neuroanatomical lesion in Vietnam veterans with penetrating head injury. Percept Mot Skills 71:1120-1122.

Heilman KM, Watson RT, Valenstein E, Goldberg ME (1987) Attention: behavior and neuronal mechanisms. In: The handbook of physiology, Sec 1, The nervous system (Plum F, Mountcastle VB, Beiger ST, eds), pp 461-481. Bethesda, MD: American Physiological Society.

Jonides J, Smith EE, Koeppe RA, Awh E, Minoshima S, Mintun MA (1993) Spatial working memory in humans as revealed by PET. Nature 363:623-625.

Markowitsh HJ, Pritzel M (1985) The neuropathology of amnesia. Prog Neurobiol 25:189-287.

McCarthy G, Blamire AM, Puce A, Nobre AC, Boch G, Hyder F,
Goldman-Rakic P, Shulman RG (1994) Functional magnetic resonance imaging of human prefrontal cortex activation during a spatial working memory task. Proc Natl Acad Sci USA 91:8690-8694.

Mishkin M (1964) Perseveration of central sets after frontal lesions in monkeys. In: The frontal granular cortex and behavior (Warren JM, Akert K, eds), pp 219-241. New York: McGraw Hill.

Petrides M, Alivisatos B, Evans AC, Meyer E (1993) Dissociation of human mid-dorsolateral from posterior dorsolateral frontal cortex in memory processing. Proc Natl Acad Sci USA 90:873-877.

Rizzolatti G, Camarda R (1987) Neural circuits for spatial attention and unilateral neglect. Amsterdam: Elsevier.

Shallice T, Burgess PW (1991) Deficits in strategy application following frontal lobe damage in man. Brain 114:727-741.

Smith EE, Jonides J, Koeppe RA, Awh E, Schumacher EH, Minoshima S (1995) Spatial versus object working memory: PET investigations. J Cognit Neurosci 7:337-356.

Stuss DT, Gow CA, Hetherington CR (1992) "No longer Gage": frontal lobe dysfunction and emotional changes. J Consult Clin Psychol 60:349-359.

Swartz BE, Halgren E, Fuster JM, Simpkins F, Gee M, Mandelkern M (1995) Cortical metabolic activation in humans during a visual memory task. Cereb Cortex 3:205-214.

Tranel D (1996) The Iowa-Benton school of neuropsychological assessment. In: Neuropsychological assessment of neuropsychiatric disorders (Grant I, Adams KM, eds), pp 81-101. New York: Oxford UP.

Tranel D, Anderson SW, Benton AL (1994) Development of the concept of "executive function" and its relationship to the frontal lobes. In: Handbook of neuropsychology (Boller F, Grafman J, eds), pp 125-148. Amsterdam: Elsevier.

Wilson FAW, Scalaidhe SPO, Goldman-Rakic PS (1993) Dissociation of object and spatial processing domains in primate prefrontal cortex. Science 260:1955-1958. 